\title{
Perturbed Lane-Emden Equations as a Boundary Value Problem with Singular Endpoints
}

\author{
Radosław Antoni Kycia ${ }^{1,2}$
}

Received: 22 October 2018 / Published online: 14 May 2019

(C) The Author(s) 2019

\begin{abstract}
The paper presents the solution for the existence of analytic solutions for some generalized Lane-Emden (LE) equation. Such a solution exists on the unit interval, in which endpoints are singularities of the proposed perturbed LE equation. The solution has many possible applications and one of the examples was provided.
\end{abstract}

Keywords Generalized Lane-Emden equations · Analytic solutions · Singularities

Mathematics Subject Classification (2010) 34A34 · 34M35

\section{Introduction}

The Lane-Emden (LE) equation [3, 5, 10] is one of the simplest nonlinear equations appearing in astrophysics. It describes balance in gaseous medium described by polytropic equation, between gravity that tries to squeeze it and internal pressure that try to prevent such collapse.

It is used in astrophysics as a first approximation to the more complicated star structure models $[3,5,7,10]$ and recently for describing molecular cloud cores [22]. Some exact solutions [19] are known and are useful in solving (matching) problems in which the interior is described by LE equation and the exterior area by some other equation. Such exact solutions appear also for higher space dimensions [15] and can be used for representation of symmetric Riemannian manifold with constant curvature as conformal flat model [20].

However, apart from applications, the LE equation is the simplest nonlinear equation that arises from the radial part of the Laplace operator in flat space with a nonlinear term. Therefore, it serves also as a building block for more complicated nonlinear differential equations, e.g., nonlinear wave equation with power-type nonlinearity $[1,13]$. There are

Radosław Antoni Kycia

kycia.radoslaw@gmail.com

1 Department of Mathematics and Statistics, Masaryk Univeristy, Kotlářská 267/2, 61137 Brno, Czech Republic

2 Faculty of Physics, Mathematics and Computer Science, Cracow University of Technology, Warszawska 24, Kraków, 31-155, Poland 
various kinds of generalization for this equation described (e.g., in [8, 11, 16, 17]). These solutions pose an interesting structure of movable singularities (singularities of analytic solutions) [14, 17, 17] and some flavor of the Painlevé analysis for them was provided in [8].

The main aim of this paper is to perturb the generalized Lane-Emden equation $[4,7,16$, 17, 17]:

$$
\frac{d^{2} u(x)}{d x^{2}}+\frac{\alpha}{x} \frac{d u(x)}{d x}+\delta u(x)^{p}=0,
$$

where $\alpha$ and $\delta$ are positive real constants and $p>1$ is a natural number.

It is assumed that the perturbed equation of Eq. 1 is in the following form:

$$
\begin{gathered}
p(x) \frac{d^{2} u(x)}{d x^{2}}+q(x) \frac{d u(x)}{d x}+r(x) u(x)+\delta u(x)^{p}=0, \\
p(x)=\left(1+a_{-1} x+a_{0} x^{2}+\ldots+a_{n} x^{n+2}\right), \\
q(x)=\left(\frac{\alpha}{x}+b_{-1}+b_{0} x+\ldots+b_{n} x^{n+1}\right), \\
r(x)=\left(c_{-1} \frac{1}{x}+c_{0}+\ldots+c_{n} x^{n}\right),
\end{gathered}
$$

where $n>-1$ is a natural number, and $\alpha>0$ and $\delta \neq 0$ are real constants. The restriction imposed on the coefficients $\left\{a_{k}\right\}_{k=-1}^{n},\left\{b_{k}\right\}_{k=-1}^{n}$ and $\left\{c_{k}\right\}_{k=-1}^{n}$ will be given below. The polynomials $p, q$, and $r$ are introduced into equations as new (fixed) singularities in the complex plane, and by a suitable change of coordinates, we can assume that the closest singularity to the origin is located at $x=1$, which results in:

$$
p(x)=(1-x) \bar{p}(x)=(1-x)\left(1+\bar{a}_{-1} x+\ldots+\bar{a}_{n-1} x^{n}+\bar{a}_{n} x^{n+1}\right),
$$

where $\bar{p}(x)$ has zeros in the complex plane that are farther from the origin than 1 . The coefficient correspondence is given by $\bar{a}_{-1}=a_{-1}, a_{k}=\bar{a}_{k+1}-\bar{a}_{k}$ for $k \in\{0, \ldots, n-1\}$ and $a_{n}=-\bar{a}_{n}$.

It will be explained how to find the solution of boundary problem - find analytic solution(s) which connect these two fixed singularities at $x=0$ and $x=1$. The procedure that constructs such kind of analytic solutions and therefore provides a solution for this nonlinear (singular) boundary value problem will be the main topic of this paper.

This problem can be used to construct solutions of Eq. 2 on the unit interval and match it for some $x>1$ with a solution to some other equation due to analyticity and therefore it is important in applications.

By deformation of the unit interval onto some smooth curve in the complex plane we will get a general problem of finding analytic solutions on such a curve that connects two (not necessary closest) fixed singularities of the deformed equation (1). The assumption on the smoothness of the curve is important as we do not want to introduce additional singularities along it.

Equation 2 is general enough to describe many problems that arise in mathematical physics. For example, the equation:

$$
\left(1-x^{2}\right) \frac{d^{2} u(x)}{d x^{2}}+\left(\frac{\alpha}{x}+\beta x\right) \frac{d u(x)}{d x}-\gamma u(x)+\delta u(x)^{p}=0,
$$

appears in $[1,13,14]$ for some special values of parameters as the equation for self-similar (analytic) profiles of nonlinear wave equation in flat spacetime on the unit interval. In this paper, the general theory for such a kind of equations will be provided and Eq. 4 will be analyzed as an example for real parameters: $\alpha>0, \beta, \gamma$ and $\delta \neq 0$.

The paper is organized as follows: In the next section, the existence of some special singular solution at $x=0$, which is a crucial ingredient for global analytic solution on the interval $[0 ; 1]$, will be given, and then the local existence of the analytic solutions around the singular point $x=0$ and $x=1$ will be proved. Then, the next section presents the general discussion of the existence of global solutions on the unit interval. Finally, an example of 
the application of the results of the paper will be presented in Eq. 4. In Appendix, some technical results are collected for the reader's convenience.

\section{Local Existence}

\subsection{Local Solution at $x=0$}

For the existence of the global analytic solutions on the unit interval, the following singular solution at $x=0$ will be of paramount importance:

$$
u_{\infty}(x)=b_{\infty} x^{a}, \quad b_{\infty}=\left(\frac{2[\alpha(p-1)-(p+1)]}{\delta(p-1)^{2}}\right)^{1 /(p-1)}, \quad a=\frac{1}{1-p} .
$$

Therefore, first, the class of Eq. 2 which has the solution (5) is singled out by the following:

Proposition 1 If Eq. 2 allows the solution (5) then the coefficients have to fulfill the following conditions:

$$
a(a-1) a_{k}+a b_{k}+c_{k}=0, \quad k \in\{-1, \ldots, n\},
$$

where a is defined in Eq. 5.

Proof The proof is straightforward. Substituting (5) into (2) and collecting terms of the same order in $x$, we obtain:

$$
\left[b_{\infty}(a(a-1)+\alpha a)+\delta b_{\infty}^{p}\right] x^{a-2}+\sum_{k=-1}^{n} b_{\infty}\left[a(a-1) a_{k}+a b_{k}+c_{k}\right] x^{a+k}=0 .
$$

The term at $x^{a-2}$ vanishes, which is exactly the definition of $b_{\infty}$. In addition, if Eq. 5 is the solution of Eq. 2, then all the other coefficient terms have to vanish, which gives the condition (6).

Hereafter, we assume that Eq. 2 fulfills (6), and therefore (5) is a solution.

The next proposition shows the existence of local analytic solutions at $x=0$ for Eq. 2 and their behavior for large initial data $u(0)=c$.

Proposition 2 There exist analytic solutions of Eq. 2 at $x=0$ with initial data $u(0)=c$.

For large $c$, the asymptotic of this analytic solution of Eq. 2 at fixed $x_{0}$ within its radius of convergence is described by scaled asymptotics of the generalized Lane-Emden equation, namely (see also Eq. 46 in Appendix):

$$
u_{ \pm}\left(x_{0}, c\right) \approx b_{\infty} x_{0}^{-2 /(p-1)}\left(1 \pm A_{0} c^{-\frac{\alpha+3+p(1-\alpha)}{4}} x_{0}^{\frac{\alpha+3+p(1-\alpha)}{2(p-1)}} \sin \left(\omega \ln \left(c^{\frac{p-1}{2}} x_{0}\right)+\phi\right),\right.
$$

where $p \neq p_{Q}$, odd and $f(p, \alpha)<0$, where:

$$
\begin{gathered}
p_{Q}:=\frac{\alpha+3}{\alpha-1}, \\
f(p, \alpha):=(-1+\alpha)^{2}+p^{2}\left(9-10 \alpha+\alpha^{2}\right)-2 p\left(-3-6 \alpha+\alpha^{2}\right),
\end{gathered}
$$

and where:

$$
\omega(p, \alpha)=i \frac{\sqrt{-f(p, \alpha)}}{2(p-1)} .
$$


Here, $A_{0}$ and $\phi$ are constants.

Proof First, we have to prove that there exists a local analytic solution at $x=0$. If we rewrite (2) in the form:

$$
\left\{\begin{array}{l}
x u^{\prime}=x v \\
x v^{\prime}=\frac{-1}{1+\sum_{k=-1}^{n} a_{k} x^{k+2}}\left(\alpha+v \sum_{k=-1}^{n} b_{k} x^{k+2}+u \sum_{k=-1}^{n} c_{k+1} x^{k+1}+x \delta u^{p}\right)
\end{array}\right.
$$

The second equation has the following form:

$$
x v^{\prime}=-\alpha v+x g(x, u, v),
$$

where $g$ is analytic at $x=0$. Therefore, using Proposition 5 from Appendix, we conclude that there is an analytic solution at $x=0$ expressible in a power series form, in which the first term is initial data $c=u(0)$. It can be found by a formal procedure of substituting a formal power series $u(x)=\sum_{k=0}^{\infty} h_{k} x^{k}$ into Eq. 2 and obtaining recurrence for the coefficients $h_{k}$ of this series. Then, the above statement assures us that this formal series is convergent-it is a solution.

For proving the asymptotic (8), Eq. 2 will be transformed into the generalized LaneEmden (1) and Proposition (7) of Appendix will be used. We use the substitution from [1, 13]:

$$
u=c w, \quad y=c^{\gamma} x, \quad \gamma=(p-1) / 2
$$

which transform Eq. 2 into $\left('=\frac{d}{d y}\right)$

$$
w^{\prime \prime}+\frac{\alpha}{y} w^{\prime}+\delta w^{p}=-\frac{1}{c^{p}}\left(w^{\prime \prime} \sum_{k=-1}^{n} a_{k} \frac{y}{c^{k \gamma+1}}+w^{\prime} \sum_{k=-1}^{n} b_{k} \frac{y^{k+1}}{c^{k \gamma+1}}+\sum_{k=-1}^{n} c_{k} \frac{y^{k}}{c^{k \gamma+1}}\right) .
$$

In the limit $c \rightarrow \infty$, the RHS of Eq. 15 vanishes and we are left with the Lane-Emden (1). To make the limiting process precise, we start from the analytic solution at a point $x_{0}$ within its circle of convergence of analytic solution. Then, we perform $c \rightarrow \infty$ limit termwise which transform the analytic solution into the series solution (43) for the LE - the solution of Eq. 15 with vanishing RHS and initial condition $w(0)=1$. Using large $y$ asymptotic of the LE (1) of the form (46) from Appendix, and returning to the original variables $u$ and $x$, we obtain exactly (8), as claimed.

The asymptotic (8) is analogous to Proposition 7 of Appendix, and results from the appearance of the LE (1) as a basic building block of Eq. 2. Loosely speaking, Proposition 2 shows that when the phase plane $\left(u\left(x_{0}\right) ; u^{\prime}\left(x_{0}\right)\right)$ fixed at $x_{0}$ is considered, then the analytic solution of Eq. 2 behaves as the spiral (8) parametrized by $c$ that wraps around the limit point:

$$
P_{\infty}=\left(b_{\infty} x_{0}^{-2 /(p-1)},-\frac{2}{p-1} b_{\infty} x_{0}^{-2 /(p-1)}\right) .
$$

The same behavior shows the special case of Eq. 2 and it was described in [1, 13].

One can note that the proof does not depend on the condition (6).

An obvious Corollary for the Proposition 2 is:

Corollary 1 For a large $u(0)=c$ value, the analytic solutions have movable singularities located as for the analytic solutions of the LE (1), among other movable singularities. 
Proof Since (15) for $c \rightarrow \infty$ goes to Eq. 1, therefore during this process, the movable singularities of the analytic solution of Eq. 2 approach the movable singularities of Eq. 1 described in [17], i.e., they are located symmetrically around the origin on the rays connecting the origin with all $p$ roots of -1 .

This result ends our discussion of behavior of local analytic solutions at $x=0$. We now pass on to the existence of the local solution at $x=1$.

\subsection{Local Existence at $x=1$}

In order to consider the local analytic solutions around $x=1$, the new variable $y=1-x$ is introduced. Equation 2 has now the form:

$$
\begin{gathered}
y \bar{p}(y) \frac{d^{2} u(y)}{d y^{2}}-q(y) \frac{d u(y)}{d y}+r(y) u(y)+\delta u(y)^{p}=0, \\
\bar{p}(y)=\left(A+A_{-1} y+A_{0} y^{2}+\ldots+A_{n} y^{n+2}\right), \\
q(y)=\left(\frac{\alpha}{1-y}+B_{-1}+B_{0} y+\ldots+B_{n} y^{n+1}\right), \\
r(y)=\left(c_{-1} \frac{1}{1-y}+C_{0}+\ldots+C_{n} x^{n}\right),
\end{gathered}
$$

where $A=1+\sum_{l=-1}^{n} a_{l}$ and $B_{-1}=b_{-1}-\sum_{l=0}^{n} b_{n}$.

On substituting formal powers series $u(y)=\sum_{l=0}^{\infty} d_{l} y^{l}$ and using the well-known Cauchy formula (42) from Appendix, the recurrence for $d_{l}$ coefficients is obtained:

$$
\begin{gathered}
d_{0}=b, \\
(l+1)(A l-B) d_{l+1}=f_{l+1}\left(d_{0}, \ldots, d_{l}\right), \quad l>-1,
\end{gathered}
$$

where, as before, $A=1+\sum_{l=-1}^{n} a_{l}, B=\alpha+B_{-1}$ and $f_{l+1}$ is a polynomial function and $b=u(y=0)$ is the initial data at $x=1$. Defining:

$$
k=\frac{B}{A},
$$

we have the following two cases:

1. $k<0$ or $k>0$, noninteger: There is infinite recurrence relations for the coefficients $d_{l}$;

2. $k \in \mathbf{N}$ (resonance condition): There is formal solution of the form $u(y)=d_{0}+\ldots+$ $d_{k} y^{k}+d_{k+1}\left(d_{0}, \ldots, d_{k}, b\right) y^{k+1}+\ldots$, where the coefficients $\left\{d_{l}\right\}_{l=k+1}^{\infty}$ are derived from the recurrence relation and the first $k+1$ coefficients have fixed values that result from the set of polynomial equations:

$$
\left\{\begin{array}{c}
f_{0}\left(d_{0}\right)=d_{0}\left(C+d_{0}^{p-1}\right)=0 \\
f_{1}\left(d_{0}, d_{1}\right)=0 \\
\ldots \\
f_{k}\left(d_{0}, \ldots, d_{k}\right)=0
\end{array}\right.
$$

where $C=c_{-1}+C_{0}$. In principle, such a system can have complex-valued solutions.

The next step is to prove that these formal solutions are in fact solutions, i.e., they are convergent. It is immediate for finite-form solutions of the second case (when $b$ is selected in such a way that $d_{k+1}=0$ ). The structure of the formal solutions is similar to those for Eq. 4 with some specially selected values of parameters, and the convergence of this special case was given in the Appendix of [13]. Therefore, the proof for the convergence of formal power series of Eq. 17 is similar. The only difference is the multiplicity of coefficients. Therefore, we have: 
Proposition 3 Formal analytic solutions of Eq. 17 at $y=0$ obtained above are in fact the solutions, i.e., they are convergent in some neighborhood of $y=0$.

Proof The idea of the proof is to transform (17) into the form required by Proposition 5 from [2] cited in Appendix. The proof is similar to those in the Appendix of [13] and therefore the sketch is only provided.

The proof depends on the value of $k$.

For $k<0$, Eq. 17 can be transformed into:

$$
\left\{\begin{array}{c}
y u^{\prime}=y v \\
y v^{\prime}=k v-C u-\delta u^{p}+y f(y, u, v),
\end{array}\right.
$$

where $f(y, u, v)$ is some analytic function of arguments and $k$ is given by Eq. 19 . Introducing new variable $\tilde{v}$ via:

$$
v=\tilde{v}+\frac{1}{k C} u+\frac{\delta}{k} u^{p},
$$

we get:

$$
\left\{\begin{array}{c}
y u^{\prime}=y g(y, u, v) \\
y v^{\prime}=k v+y \tilde{f}(y, u, v),
\end{array}\right.
$$

where again $g$ and $\tilde{f}$ are some analytic functions. Since $k<0$, we can apply the aforementioned proposition which guarantees convergence of formal power series.

For non-integer $\llcorner k\lrcorner<k<\ulcorner k\urcorner$, the general idea of the proof is as follows. First, the new function $w(y)$ via:

$$
u(y)=d_{0}+\ldots+\left(d_{\llcorner k\lrcorner}+y w(y)\right) y^{\llcorner k\lrcorner},
$$

is defined, where $\left\{d_{l}\right\}_{l=0}^{\llcorner k\lrcorner}$ coefficients are given by the recurrence relation (18). Then, switching to the first order system of ODEs, removing constant terms, and diagonalizing linear parts, we arrive into the system required by Proposition 5 .

The last part of the proof is also valid for $k \in \mathbf{N}$.

The idea of the proof resembles the transformation to the normal form [12, 21]. As for $x=0$, one can note that the proof does not depend on the condition (6) and therefore it is a general result for Eq. 2 .

In the next section, a general discussion on the existence of global analytic solutions on unit interval will be given.

\section{Global Existence of Analytic Solutions}

In order to get global solution, generalization of the method proposed in [1] and developed in [13] will be used. For such general class as Eq. 2, we present only steps that can be done and not a strict algorithm - these steps have to be adjusted to the concrete equation under consideration. The steps are as follows:

- Construct local analytic solutions at $x=0$ (existence proved in Proposition 2) and prove that they can be extended toward $x=1$.

- Construct local analytic solutions at $x=1$ (existence proved in Proposition 3) and prove that they can be extended toward $x=0$.

- Match theses local solutions at some point inside the unit interval to obtain the global solution. 
For proving that there is no singularities along the unit interval, one can use some global methods, e.g., Lyapunov function (see [6, 21] for general reference on Lyapunov functions). If this is proved, then the local analytic solution can be extended along the real axis from $x=0$ toward $x=1$. Usually, the similar proof can be performed for the local analytic solution that starts from $x=1$, and it can be shown that it can be extended to $x=0$ along the real axis.

The proof of analytic continuation of local analytic solutions will be provided for a restricted class of Eq. 2, namely, we have:

Proposition 4 For $\left(\frac{p^{\prime}(x)}{2}-q(x)\right)<0, p(x)>0$ in $x \in[0 ; 1]$, a constant $r(x)=r$, and odd p, the local analytic solutions of Eq. 2 around $x=0$ and $x=1$ described in the previous section can be extended along the unit interval, i.e., there is no (movable) singularities of the extended solutions in $[0 ; 1]$.

The assumption $p(x)>0$ in the unit interval is not restrictive since the whole equation can be multiplied by -1 . There is $p(x) \neq 0$ in the unit interval since there is no (fixed) singularity of Eq. 2 in this interval by assumption.

Proof The proof goes along the same line as in $[1,13]$ and based on the Lyapunov function

$$
H(x)=\frac{p(x)}{2} u^{\prime}(x)^{2}+\frac{\delta}{p+1} u(x)^{p+1}+\frac{r}{2} u(x)^{2} .
$$

Its derivative on the solutions of Eq. 2 is:

$$
H^{\prime}=\left(\frac{p^{\prime}(x)}{2}-q(x)\right) u^{\prime 2}<0 .
$$

Since $H$ is monotone on solutions, therefore denoting $V_{\min }=\min _{u}\left(\frac{\delta}{p+1} u^{p+1}+\frac{r}{2} u^{2}\right)(p$ is odd and therefore global minimum exists), we have from $H(x) \leq H(0)$ the following estimate:

$$
\frac{p(x)}{2} u^{\prime}(x)^{2}<H(0)-V_{\text {min }},
$$

and therefore, since $p(x)>0$ in $[0 ; 1]$, we get that $\left|u^{\prime}(x)\right|$ is bounded and the analytic solution extended from $x=0$ toward $x=1$ is also bounded.

For extension of the analytic solution at $x=1$ toward 0 , the following inequality will be used:

$$
\frac{-H^{\prime}}{1+H} \leq-\frac{p^{\prime}(x)-2 q(x)}{p(x)} .
$$

Integrating from $x=1$ to some $0<\bar{x}<<1$ and using the fact that the RHS is regular in this interval, we get that $H$ is bounded and therefore $u$ and $u^{\prime}$ are finite as well.

In the last step, analytic solutions extended from both endpoints have to be matched at some intermediate point $x_{0} \in[0 ; 1]$, as is presented in Fig. 1 .

The matching can be intuitively visualized when we analyze the situation on the phase plane at $x_{0}$. Figure 2 presents the situation.

If we will vary $c$ initial data of the solution starting from $x=0$ then, as Proposition 2 states, we obtain the spiral that wraps around $P_{\infty}$, which is $C_{0}$ curve. The second curve $C_{1}$ is the image of the solution extended from $x=1$, and it is parametrized by $u(1)=b-$ the initial data at this endpoint. Each intersection of these curves is exactly $C^{1}$ matching condition; moreover, it is analytical matching, because we match two analytic solutions. 


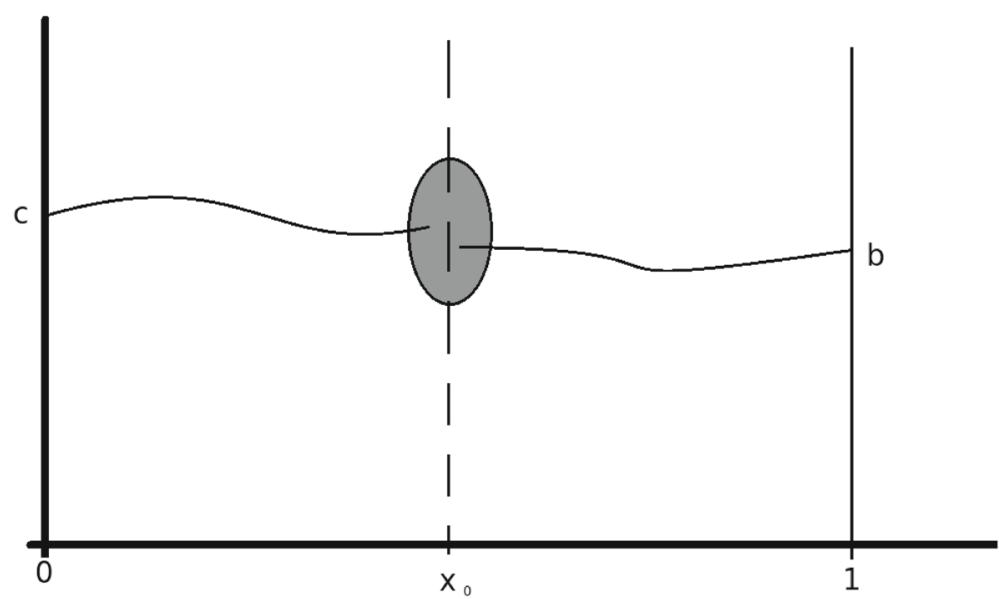

Fig. 1 Matching of two local analytic solutions extended to $x_{0}$ point

Going along $C_{1}$ curve, we start from $b=0$ - the trivial solution. Increasing $b$, we can get a few possibilities for the number of intersections:

1. In the first case, the $C_{1}$ curve passes exactly through the wrapping point of the spiral$P_{\infty}$. In this case, we obtain countable family of analytic solutions on unit interval (case 1 in the figure). For this case, the existence of $u_{\infty}$ solution, i.e., the condition (6), is required. It is obvious to explain why it is the case if we use continuous dependence of solutions on initial conditions with initial data that are slightly shifted from singular points $x=0$ and $x=1$, which however match these analytic solutions for some initial data specified at these singular points. The $x=0$ solution "wraps around" (5). The

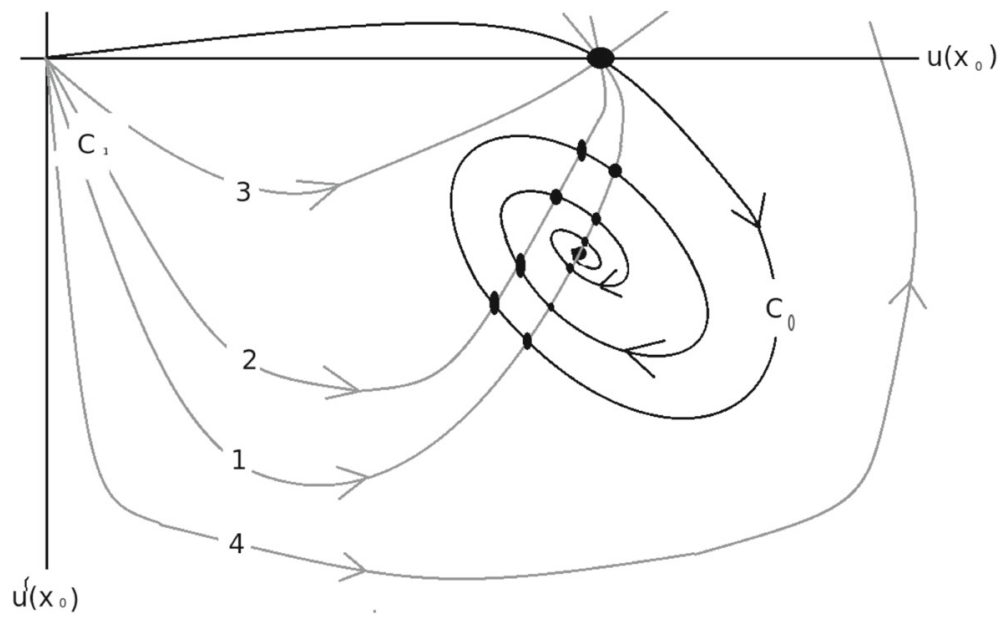

Fig. 2 Matching of two local analytic solutions extended to $x_{0}$ point. The cases 1,2 , and 3 belong to the same type of the equation with different values of coefficients. Case 4 , topologically different from the previous ones, corresponds to the different equation. Wrapping point of $C_{0}$ curve moves when the values of the equation's coefficients are changing 
second solution from $x=1$ stays close to this point if we vary initial data $b$ around $b_{\infty}$ value, therefore, also around (5).

2. The second curve can cross $C_{0}$ in a finite number of points-a finite number of global solutions (case 2 in the figure). In this case, the condition (6) is slightly perturbed.

3. If there are some special solutions, like constant ones for the case of resonances for $x=1$ solution (see also the next section for the example), then the second curve can cross $C_{0}$ at one point which corresponds to this special solution (case 3 in the figure).

4. The second curve can also have only one point $(0 ; 0)$ (which corresponds to the trivial solution) with the $C_{0}$ curve (case 4 in the figure).

The statements of this qualitative discussion will now be explained in the example in the following section.

\section{Example}

In this section, a simple example (4) that illustrates the above results and discussion is presented. It is a generalization of the equation analyzed in $[1,13,14]$. The other example of the class (2) is discussed in [18].

It is assumed that $\alpha>0, \beta, \gamma$ and $\delta \neq 0$ are real parameters for Eq. 4 .

The condition for the existence of the solution (5) is of the form:

$$
\gamma=\frac{2}{1-p}\left(\frac{p+1}{p-1}+\beta\right),
$$

and it is assumed that it is hereafter fulfilled. In addition, there exists the constant solution:

$$
u_{0}=\left(\frac{\gamma}{\delta}\right)^{1 /(p-1)}:=b_{0},
$$

providing that $\gamma>0(\delta>0$ was assumed previously $)$.

We start from analyzing local power series solution at $x=0$, in which Proposition 2 assures to exist. On substituting formal power series $u(x)=\sum_{k=0}^{\infty} a_{k} x^{k}$ into Eq. 4, we get unique recurrence for the coefficients:

$$
a_{0}=c=\text { arbitrary, } \quad a_{1}=0, \quad a_{k+2}=\frac{\left[(k(k-1-\beta)+\gamma] a_{k}-\delta c_{k}\right.}{(k+2)(k+1+\alpha)},
$$

where $k \geq 0$ and $c_{k}$ can be computed from $b_{k}$ ones using Eq. 42 formula. This power series can be analytically continued from $x=0$ to the vicinity of $x=1$ along the real line without encountering any singularities. It can be proved by repeating the proof of Proposition 4 with additional assumptions, that $p$ is odd, and $\delta>0$. To this end, we use the following Lyapunov function:

$$
H\left(u, u^{\prime} ; x\right)=\left(1-x^{2}\right) \frac{u^{\prime 2}}{2}-\gamma \frac{u^{2}}{2}+\frac{\delta}{p+1} u^{p+1} .
$$

We can shift this function by a constant (the minimum of polynomial in $u$ consisting of the last two terms) to make it positively defined. Taking derivative and replacing $u^{\prime \prime}$ with the help of Eq. 4, we obtain:

$$
H^{\prime}=-\left((1+\beta) x+\frac{\alpha}{x}\right) u^{\prime 2} \text {. }
$$

the term in the bracket is positive on the unit interval when $\beta>-1$ or $\beta=0$ or when $\beta<-1$ and $\alpha+\beta>-1$. Assuming this, we have that $H^{\prime}<0$, therefore, $H(0)>H(x)$ for 
$0<x<1$. From this, we obtain immediately, as in [1,13], that solution which is regular at $x=0$ stays finite along the unit interval.

The solution at $x=1$ can be obtained by introducing the variable $y=1-x$ into Eq. 4 , to obtain $\left({ }^{\prime}=d / d y\right)$ :

$$
y(2-y) u^{\prime \prime}-\left(\frac{\alpha}{1-y}+\beta(1-y)\right) u^{\prime}-\gamma u+\delta u^{p}=0 .
$$

Introducing a power series ansatz $u(y)=\sum_{k=0}^{\infty} b_{k} y^{k}$, we obtain the following recurrence for the coefficients:

$$
\begin{aligned}
& b_{0}=b=\text { arbitrary, } \quad b_{1}=\frac{\gamma b_{0}-\delta c_{0}}{-(\alpha+\beta)}, \\
& b_{k+1}=\frac{k(3(k-1)-2 \beta) b_{k}+\gamma b_{k}-\delta c_{k}+(k-1)(\beta+2-k) b_{k-1}-\gamma b_{k-1}+\delta c_{k-1}}{(k+1)(2 k-\alpha-\beta)},
\end{aligned}
$$

where $c_{k}$ can be computed from $b_{k}$ ones employing (42) formula. When $\alpha+\beta=2 k$, where $k \geq 0$ is a natural number, we obtain the resonance condition, which leads, as in [13], to the special class of the solutions described in Section 2.2. We will not discuss this class as it is analogous to the analysis in [13]. Therefore, we assume that $-1<\alpha+\beta<0$.

The convergence proof for the solution with the coefficients (35) is given by Proposition 3 ; however, it is instructive to provide the special case of this proof. To prove that this

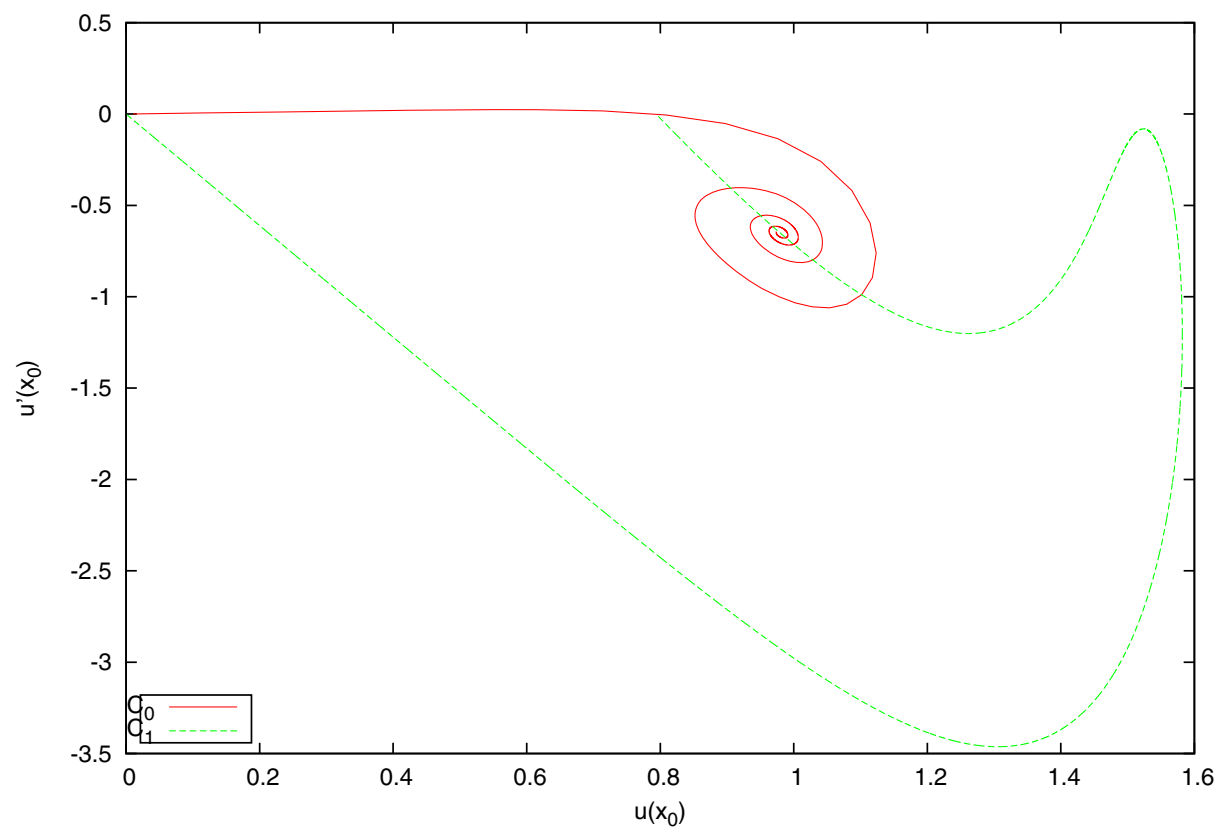

Fig. 3 Plot for $\alpha=2, \beta=-25 / 12, \gamma=1 / 4, \delta=1$. In this case, matching point $x_{0}=0.5$, and wrapping point $P_{\infty}=\left(\frac{\sqrt{2}}{\sqrt[3]{3}} ;-\frac{2 \sqrt{2}}{3 \sqrt[3]{3}}\right)$. The curve starts from $(0 ; 0)$ point and passes through $P_{\infty}$ and integration was terminated at $\left(b_{0} ; 0\right)$ point 
power series has nonzero radius of convergence, we rewrite it as a system of first-order ODEs:

$$
\left\{\begin{array}{l}
y u^{\prime}=y v \\
y v^{\prime}=\frac{1}{(2-y)(1-y)}\left(\left(\alpha+\beta\left(1+y^{2}-2 y\right)\right) v+\gamma(1-y) u-\delta(1-y) u^{p} .\right.
\end{array}\right.
$$

We are allowed to use Proposition 5 if we remove the terms of $u$ and $u^{p}$ with constant coefficients. This can be done using the following change of variables:

$$
v=\bar{v}-\frac{\gamma}{\alpha+\beta} u+\frac{\delta}{\alpha+\beta} u^{p}
$$

which transforms (36) into:

$$
\left\{\begin{array}{l}
y u^{\prime}=y f(y, u, v) \\
y v^{\prime}=2(\alpha+\beta)+y g(y, u, v)
\end{array}\right.
$$

where $f$ and $g$ are functions analytic in all of its variables around $y=0$. From the fact that $\alpha+\beta<0$ and Proposition 5, we conclude that the solution (35) is convergent in some neighborhood of $y=0(x=1)$.

When $2 k<\alpha+\beta<2(k+1)$ or when a resonance condition occurs, namely $\alpha+\beta=2 k$, for natural $k$, then the general proof is a simple modification of those from the Appendix of [13].

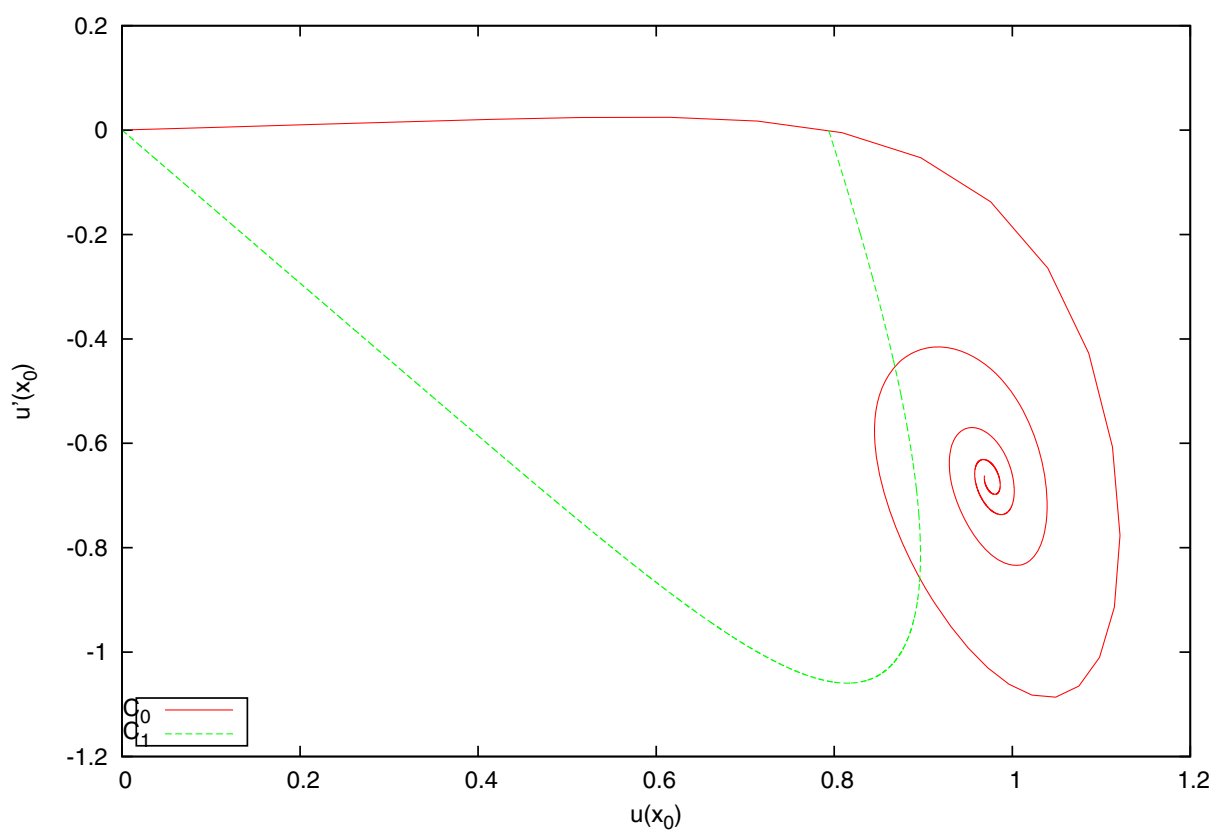

Fig. 4 Plot for $\alpha=2, \beta=-29 / 12, \gamma=1 / 4, \delta=1$. In this case, the matching point $x_{0}=0.5$, and wrapping point $P_{\infty}=\left(\frac{\sqrt{2}}{\sqrt[3]{3}} ;-\frac{2 \sqrt{2}}{3 \sqrt[3]{3}}\right)$. The curve starts from $(0 ; 0)$ point but does not pass through $P_{\infty}$ as condition (29) is not fulfilled. Integration was terminated again at $\left(b_{0} ; 0\right)$ point 
In next step, we show that the solution form $x=1$ can be extended along the real line to $x=0$. We use, as in [1] and [13], the following easy to check bound:

$$
\frac{-H^{\prime}}{H+1}<\frac{2 \alpha}{x}
$$

from which it occurs, by integrating both sides, that $u$ and $u^{\prime}$ are bounded inside the unit interval if we start from solution (35) bounded at $x=1$.

These results assure us that these two local solutions can be matched at some $x_{0}$ inside the unit interval. This matching fits exactly the general scheme presented in the previous section: On the phase plane at $x_{0}$, the solution form $x=0$ gives a spiral which wraps around (5) solution. The second curve for solution at $x=1$ crosses this spiral, such that every intersection gives a global solution. We can note that there will be at least two such intersections, which correspond to the $u=0$ and $u_{0}$ solution.

Figure 3 presents the case with many countable global solutions. For this case, condition (29) is fulfilled. If we now slightly perturb the equation coefficients that this condition is not fulfilled, then we get a finite number of intersections/global solutions, and in the end we are left only with two constant solutions. This is presented in Figs. 4 and 5.

This example shows that for the specific case presented in $[1,13]$ the coefficients appear perfectly to fulfill the matching condition (29).

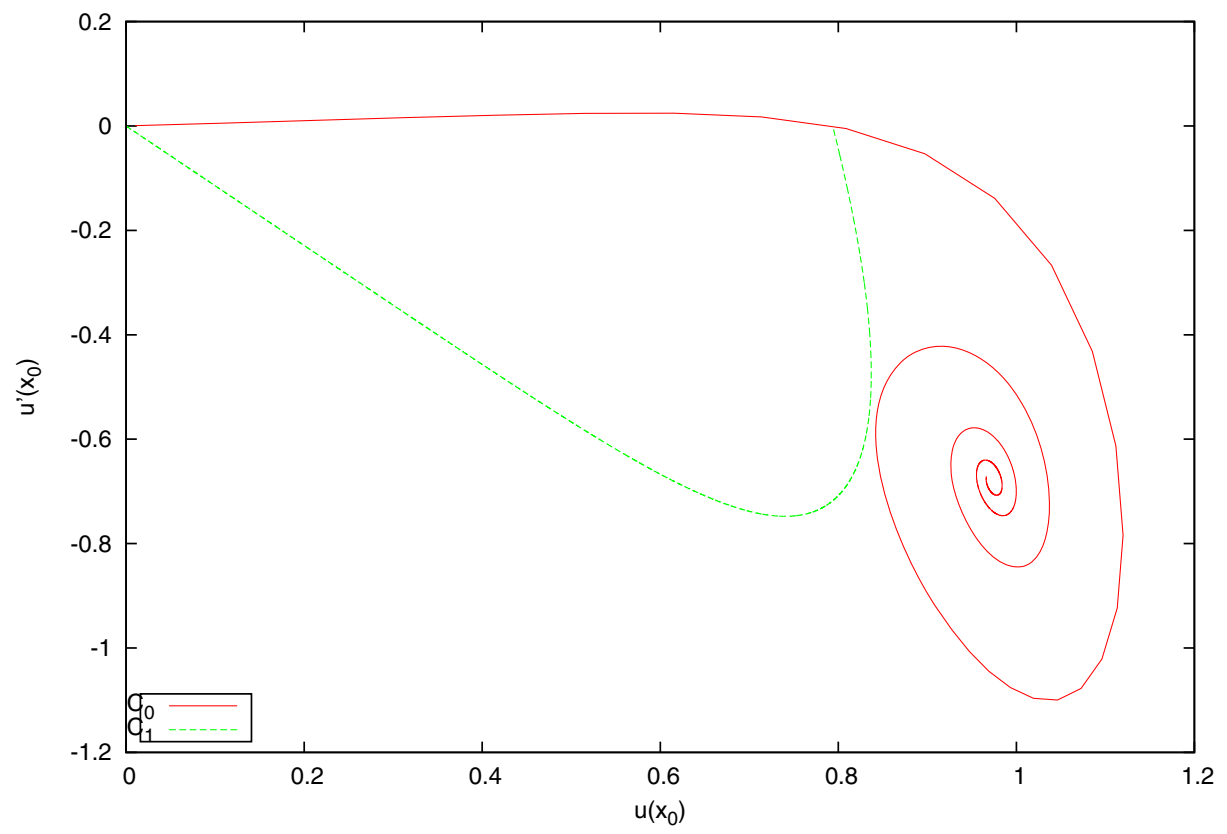

Fig. 5 Plot for $\alpha=2, \beta=-31 / 12, \gamma=1 / 4, \delta=1$. In this case, the matching point $x_{0}=0.5$, and wrapping point $P_{\infty}=\left(\frac{\sqrt{2}}{\sqrt[3]{3}} ;-\frac{2 \sqrt{2}}{3 \sqrt[3]{3}}\right)$. The curve starts from $(0 ; 0)$ point but does not pass through $P_{\infty}$ as condition (29) is not fulfilled. Integration was terminated again at $\left(b_{0} ; 0\right)$ point. Now, we only get two global solutions-the trivial one and $u_{0}$ 


\section{Conclusions}

A general method of matching using a singular solution at one of the fixed singularity of the second-order differential equation that generalizes the Lane-Emden equation was presented. The method is constructive and shows under what circumstances countable the family of analytic solutions that interpolate between fixed singularities of the equation exists. This is a significant generalization of the idea presented in [1] and [13].

This theory was used to describe a matching problem for the differential equation generalizing the equations for self-similar profiles of nonlinear wave equations with power-type nonlinearity. This example sheds a new light on the special case presented in [13].

The boundary value problem and equations of the type presented in the paper are very common in applications since they arise from the radial part of the Laplace operator and power-type nonlinearity; therefore, these results are useful in applied sciences and technical problems.

Funding Information This research was supported by the GACR grant 17-19437S, and the grant MUNI/A/1138/2017 and MUNI/A/1186/2018 of Masaryk University. We also thank the PHAROS COST Action (CA16214) for partial support.

Open Access This article is distributed under the terms of the Creative Commons Attribution 4.0 International License (http://creativecommons.org/licenses/by/4.0/), which permits unrestricted use, distribution, and reproduction in any medium, provided you give appropriate credit to the original author(s) and the source, provide a link to the Creative Commons license, and indicate if changes were made.

\section{Appendix: Preliminary facts}

This section collects some results useful in the course of the paper and scattered in the literature. They were collected for the reader's convenience.

In the paper to get the existence of the local analytic solutions around finite fixed singularities, Proposition 1 from [2] is employed, which reads:

Proposition 5 [2] Consider a system of differential equations for $i+j$ functions $u=$ $\left(u_{1}, \ldots, u_{i}\right)$ and $v=\left(v_{1}, \ldots, v_{j}\right)$,

$$
t \frac{d u_{l}}{d t}=t^{\mu_{l}} f_{l}(t, u, v), \quad t \frac{d v_{l}}{d t}=-\lambda_{l} v_{l}+t^{\nu_{l}} g_{l}(t, u, v)
$$

with constants $\lambda_{l}>0$ and integers $\mu_{l}, v_{l} \geq 1$ and let $U$ be an open subset of $R^{n}$ such that the functions $f$ and $g$ are analytic in a neighborhood of $t=0, u=c, v=0$ for all $c \in U$. Then, there exists an $i$-parameter family of solutions of the system (40) such that:

$$
u_{l}(t)=c_{l}+O\left(t^{\mu_{l}}\right), \quad v_{l}(t)=O\left(t^{\nu_{l}}\right)
$$

where $u_{l}(t)$ and $v_{l}(t)$ are defined for $c \in U,|t|<t_{0}(c)$ and are analytic in $t$ and $c$. 
In derivation of the recurrence for the equations with power-type nonlinearities, it is convenient to use the well-known Cauchy product [9]:

$$
\begin{aligned}
& \left(\sum_{l=0}^{\infty} a_{l}\left(x-x_{0}\right)^{l}\right)^{p}=\sum_{l=0}^{\infty} c_{l}\left(x-x_{0}\right)^{l}, \\
& c_{0}=a_{0}^{p}, \quad c_{m}=\frac{1}{m a_{0}} \sum_{l=1}^{m}(l p-m+l) a_{l} c_{m-l},
\end{aligned}
$$

for $m>0$, and where $a_{0}=c$ is a free parameter. The Cauchy product allows us to deal with the nonlinear term in a simple manner.

Some facts from the theory of Eq. 1 are presented following [16, 17]. Equation 1 has an analytic solution around the fixed singularity $x=0$

Proposition 6 [16] Equation 1 has a local analytic solution around the fixed singularity at $x=0$ of the form:

$$
a_{0}=\text { initial data }, \quad a_{1}=0, \quad a_{k+2}=\frac{\delta c_{k}}{(k+2)(k+1+\alpha)} \quad k \geq 0 ;
$$

The $\left\{c_{k}\right\}_{k=0}^{\infty}$ are coefficients derived from $\left\{a_{k}\right\}_{k=0}^{\infty}$ using Eq. 42 .

The proof can be found in [16].

Equation 1 has also the singular solution (5). It is singular at the origin and it somehow attracts solutions that vanish at infinity. The precise meaning is given by the following Proposition which is a generalization of the results from $[1,13]$.

Proposition $7[7,17]$ For $p \neq p_{Q}$, odd and $f(p, \alpha)<0$, where:

$$
\begin{gathered}
p_{Q}:=\frac{\alpha+3}{\alpha-1}, \\
f(p, \alpha):=(-1+\alpha)^{2}+p^{2}\left(9-10 \alpha+\alpha^{2}\right)-2 p\left(-3-6 \alpha+\alpha^{2}\right)
\end{gathered}
$$

the asymptotic of analytic solution (43) with $u(0)=a_{0}=1$ is given in the following form:

$$
u_{ \pm}(x) \approx b_{\infty} x^{-2 /(p-1)}\left(1 \pm A_{0} x^{\frac{\alpha+3+p(1-\alpha)}{2(p-1)}} \sin (\omega \ln (x)+\phi)\right.
$$

for large $x$, where:

$$
\omega(p, \alpha)=i \frac{\sqrt{-f(p, \alpha)}}{2(p-1)}
$$

and $A_{0}$ and $\phi$ are constants.

The proof for $\alpha=2$ and $\delta=1$ can be found in [7] and general proof in [17].

\section{References}

1. Bizoń P, Maison D, Wasserman A. Self-similar solutions of semilinear wave equations with a focusing nonlinearity. Nonlinearity. 2007;20:2061-2074.

2. Breitenlohner P, Forgács P, Maison D. Static spherically symetric solutions of the Einstein-Yang-Mills equations. Commun Math Phys. 1994;163:141-172.

3. Chandrasekhar S. An introduction to the study of stellar structure. University of Chicago Press. 1939.

4. Davis HT. Introduction to Nonlinear Differential and Integral Equations, Dover Publications; Reprint edition. 2010. 
5. Emden VR. Gaskugeln. Leipzig: Teubner; 1907.

6. Hartman P. Ordinary Differential Equations Society for Industrial and Applied Mathematics; 2 edition. 2002.

7. Hunter C. Series solutions for polytropes and the isothermal sphere. Mon Not R Astron Soc. 2001;328:839-847.

8. Govinder KS, Leach PG. Integrability analysis of the Emden-Fowler equation. J Nonlinear Math Phys. 2007;14(3):443-461.

9. Gradshteyn IS, Ryzhik IM. Table of Integrals, Series and Products, 5th ed. New York: Academic Press; 1994.

10. Lane JH. On the Theoretical Temperature of the Sun under the Hypothesis of a Gaseous Mass Maintaining its Volume by its Internal Heat and Depending on the Laws of Gases Known to Terrestrial Experiment. Amer J Sci Arts. 1870;2(50):5774.

11. Khaliquek CM, Mahomed FM, Muatjetjeja B. Lagrangian formulation of a generalized Lane-Emden equation and double reduction. J Nonlinear Math Phys. 2008;15(2):152-161.

12. Kossovskiy I, Zaitsev D. Normal form for second order differential equations. J Dyn Control Syst. 2017;1-22. https://doi.org/10.1007/s10883-017-9380-9.

13. Kycia R. On self-similar solutions of semilinear wave equations in higher space dimensions. Appl Math Comput. 2011;217:9451-9466.

14. Kycia R. On movable singularities of self-similar solutions of semilinear wave equations, The Proceedings of the Conference 'On Formal and Analytic Solutions of Differential and Difference Equations II'. Banach Center Publ. 2012;97:59-72. https://doi.org/10.4064/bc97-0-4.

15. Kycia R, Filipuk G. On the solutions of the critical Lane-Emden equation in higher space dimensions, Proceedings CASTR 2013 : Computer Algebra Systems in Teaching and Research: 7th International Workshop. 2013. arXiv:1705.03409.

16. Kycia R, Filipuk G. On the singularities of the Emden-Fowler type equations, the Proceedings from the ISAAC 2013; Current Trends in Analysis and its Applications. Cambridge: Birkhäuser; 2015. https://doi.org/10.1007/978-3-319-12577-0_13.

17. Kycia R, Filipuk G. On analytic solutions of the Emden-Fowler and isothermal sphere type equations. Appl Math Comput. 2015;265:1003-1010. https://doi.org/10.1016/j.amc.2015.05.140.

18. Kycia R. Towards general results on singular boundary value problem for second order ODEs with power type nonlinearity and two fixed singularities, 20th International Conference on Methods and Models in Automation and Robotics, MMAR 2015. 2015. https://doi.org/10.1109/MMAR.2015.7283871.

19. Mach P. All solutions of the $\mathrm{n}=5$ Lane-Emden equation. J Math Phys. 2012;53:062503.

20. Mach P, Murchadha N. Spherically symmetric Riemannian manifolds of constant scalar curvature and their conformally flat representations. Class Quantum Gravity. 2014;13:135001.

21. Wiggins S. Introduction to Applied Nonlinear Dynamical Systems and Chaos, 2nd ed. Berlin: Springer; 2003.

22. Yu-Qing Lou DL, Esimbek J. Lane-emden equation with inertial force and general polytropic dynamic model for molecular cloud cores. Mon Not R Astron Soc. 2018;473(2):2441-2464. https://doi.org/10. 1093/mnras/stx1925.

Publisher's Note Springer Nature remains neutral with regard to jurisdictional claims in published maps and institutional affiliations. 